\title{
Molecular Evidence of Human Monkeypox Virus Infection, Sierra Leone
}

\section{Fei Ye, ${ }^{1}$ Jingdong Song, ${ }^{1}$ Li Zhao, ${ }^{1}$ Yi Zhang, Lianxu Xia, Lingwei Zhu, Idrissa Laybohr Kamara, Jiao Ren, Wenling Wang, Houwen Tian, Guizhen Wu, Wenjie Tan}

Author affiliations: China Center for Disease Control and Prevention, Beijing, China (F. Ye, J. Song, L. Zhao, Y. Zhang, J. Ren, W. Wang, H. Tian, G. Wu, W. Tan); Sierra Leone-China Friendship Biological Safety Laboratory, Freetown, Sierra Leone (F. Ye, J. Song, Y. Zhang, L. Xia, L. Zhu, I.L. Kamara)

DOI: https://doi.org/10.3201/eid2506.180296

Monkeypox virus is a zoonotic disease endemic to Africa. In 2017, we confirmed a case of human monkeypox virus in Sierra Leone by molecular and serologic methods. Sequencing analysis indicated the virus belongs to the West African clade and data suggest it was likely transmitted by wild animals.

$\mathrm{M}$ onkeypox virus (MPXV), of the genus Orthopoxvirus, was identified in captive cynomolgus monkeys in Copenhagen in 1958 (1). The first documented case of human MPXV infection was reported in a patient from the Democratic Republic of the Congo in 1971 (2). Other outbreaks have occurred, including a large one in the United States in 2003, in which 47 confirmed and probable human cases of MPXV infection were identified after importation of wild rodents from Ghana (3). Phylogenetic analyses of MPXVs have revealed 2 distinct clades, West African and Congo Basin (4). In the past decade, human MPXV infections also have increased in Central and West Africa (5).

Ecologic niche modeling shows that Sierra Leone is in a geographic region suitable for transmission of MPXV (6). Several cases of human MPXV have been detected in West Africa, including a case in Sierra Leone in 1970 and another in March 2014 (5). We report confirmation of an MPXV infection in Sierra Leone in March 2017.

A 35-year-old man from Kpaku village, Galliness Perri chiefdom, Pujehun district, in southern Sierra Leone near the border with Liberia, sought treatment on March 16, 2017, for fever, body pain, malaise, dysphagia, and enlarged cervical lymph nodes. The patient reported hunting and eating squirrels $\approx 10$ days before becoming ill, and traveling to Pelewahun gee bu in Bo district 3 days before his symptoms began. On March 17, he began having

${ }^{1}$ These authors contributed equally to this article. generalized vesicular skin eruptions. Clinicians sent vesicular swab specimens and blood samples collected on March 28 (12 days after the patient sought treatment) and on May 10 (day 55 after the patient sought treatment) to the Sierra Leone-China Friendship Biologic Safety Laboratory in Freetown, Sierra Leone.

We conducted MPXV-specific real-time PCR, as previously described (7), and detected MPXV DNA in the day 12 blood sample (cycle threshold $=35.88$ ). In addition, we performed MPXV-specific IgG ELISA, as previously described (8). The day 12 sample was positive at 1:800 dilution and the day 55 sample was positive at 1:3,200 dilution, demonstrating a $>4$-fold increase in the MPXV-specific antibody response during the patient's recovery.

We amplified a 1,028-bp fragment of DNA for further Sanger sequencing (Figure, panel A) and submitted it to GenBank under Monkeypox virus Sierra Leone 2017 (accession no. MG906726). Phylogenetic analysis showed this strain is closer to MPXV isolates from the West African clade than from the Congo Basin clade (Figure, panel B). In addition, we found only a single nucleotide difference between the Sierra Leone 2017 fragment and MPXV Utrecht, UTC_1964 (Appendix Figure, panel A, https://wwwnc.cdc. gov/EID/article/25/6/18-0296-App1.pdf).

For further comparison, we selected previous MPXV sequences from NCBI (https://www.ncbi.nlm.nih.gov) and aligned these to Sierra Leone 2017 (Appendix Figure, panel B) using DNAMAN software (Lynnon Corporation, https://www.lynnon.com/dnaman.html). We found that Sierra Leone 2017 was closely related to UTC_1964. In addition, a 6-nucleotide deletion (GTATAC, a repeat unit) was more evident in the dUTPase region of Sierra Leone 2017 compared with other MPXVs from Africa. Also, a 25 -nucleotide insertion ( $5 \times$ repeat of TCCAT unit) in the kelch-like protein-encoding region was more evident in the USA_2003_039 genome compared with most MPXV isolates from Africa. This insertion leads the N-terminal amino acids of the protein sequence that changed from EWNGMEWNGK in USA_2003_039 to VNNFEIK in Sierra Leone 2017.

Recent studies have shown that genome-region deletion in the Congo Basin MPXV clade can affect viral replication and pathogenicity (9). The deletion we noted in Sierra Leone 2017, which is absent in previously isolated MPXVs from Africa, might be a molecular signature and further evaluation is needed to determine whether it plays a role in viral replication and pathogenicity.

The case-patient lived in a region with large areas of tropical forest and many wild mammals. An ecologic niche model suggests this region is suitable for MPXV transmission (6). Considering the patient's history of hunting and eating squirrels, and the lack of new cases among 16 identified community contacts in Pujehun, we hypothesize 
A
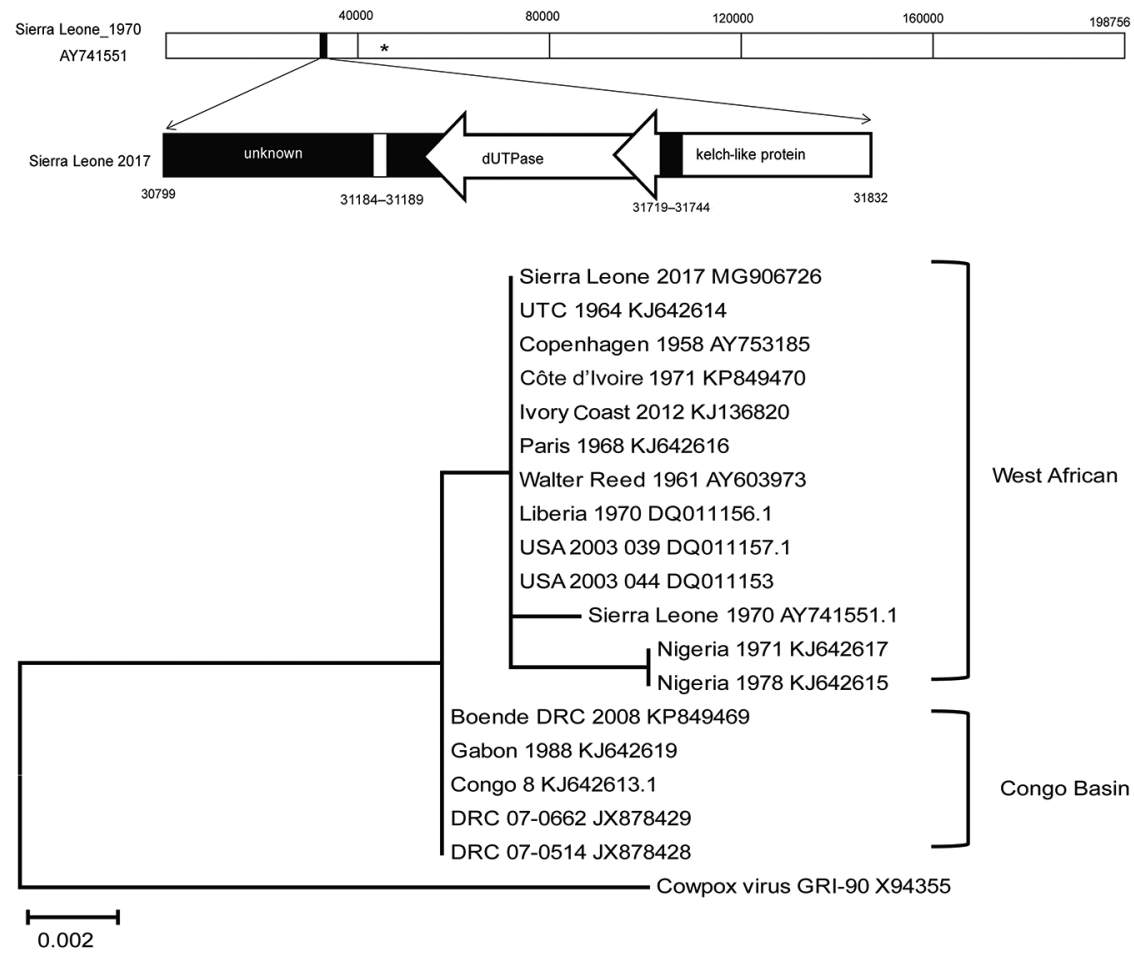

Figure. Phylogenetic analysis and molecular signatures of monkeypox virus (MPXV) Sierra Leone 2017 and other collected MPXV isolates. A) Schematic representation of the MPXV Sierra Leone 2017 genomic fragment by reference to genomic data on MPXV Sierra Leone 1970. MPXV Sierra Leone 2017 contains 3 parts: an unknown region, genes encoding dUTPase, and genes encoding partial kelchlike protein. *, binding position of primers used for real-time PCR detection. Bottom panel displays genes described. Arrows indicate direction of transcription. B) Phylogenetic relationships between genomic fragments of MPXV collected in Sierra Leone and other orthopoxviruses. Neighbor-joining phylograms constructed by using MEGA6 (https://www.megasoftware. net) and the maximum-likelihood method. Scale bar indicates nucleotide substitutions per site. that the patient was likely infected by contact with wild animals. Of note, MPXV genetic evidence from this casepatient was closely related to UTC_1964, a strain that was isolated from an animal imported from Africa to the Rotterdam Zoo in 1966 (10). Because the reservoir species of MPXV remains unknown, we hypothesize that our casepatient was probably infected by exposure to wild animals and not from MPXV imported to Sierra Leone from Central Africa. However, further serologic surveys of animals in this region could provide useful evidence for the origin of MPXV strain Sierra Leone 2017.

In summary, we described a confirmed case of human MPXV in Sierra Leone in 2017 and molecular evidence hinting of its animal origin. We suggest MPXV circulation in wild animals and humans in West Africa requires more attention, and emphasize the value of local surveillance and molecular characterization of MPXV to help determine its origin.

\section{Acknowledgment}

We thank the staff of the Ministry of Health and Sanitation, Government of Sierra Leone, for providing information on this case.

This study was supported by the National Key Research and Development Program of China (grant nos. 2016YFD0500301, 2016YFC1200905, and 2016YFC1200200), and the Megaproject for Infectious Disease Research of China (grant no. 2016ZX10004222-002).

\section{About the Author}

Dr. Ye is a scientist in the National Institute for Viral Disease Control and Prevention, Chinese Center for Disease Control and Prevention. His primary research interest is the surveillance of viral diseases, especially poxvirus and coronavirus.

\section{References}

1. Magnus PV, Andersen EK, Petersen KB, Birch-Andersen A. A pox-like disease in cynomolgus monkeys. Acta Pathol Microbiol Scand. 1959;46:156-76. http://dx.doi.org/10.1111/j.1699-0463. 1959.tb00328.x

2. Ladnyj IDZ, Ziegler P, Kima E. A human infection caused by monkeypox virus in Basankusu Territory, Democratic Republic of the Congo. Bull World Health Organ. 1972;46:593-7.

3. Reynolds MG, Davidson WB, Curns AT, Conover CS, Huhn G, Davis JP, et al. Spectrum of infection and risk factors for human monkeypox, United States, 2003. Emerg Infect Dis. 2007; 13:1332-9. http://dx.doi.org/10.3201/eid1309.070175

4. Esposito JJ, Knight JC. Orthopoxvirus DNA: a comparison of restriction profiles and maps. Virology 1985;143:230-51. https://doi.org/10.1016/0042-6822(85)90111-4

5. Durski KN, McCollum AM, Nakazawa Y, Petersen BW, Reynolds MG, Briand S, et al. Emergence of monkeypoxWest and Central Africa, 1970-2017. MMWR Morb Mortal Wkly Rep. 2018;67:306-10. http://dx.doi.org/10.15585/ mmwr.mm6710a5

6. Nakazawa Y, Emerson GL, Carroll DS, Zhao H, Li Y, Reynolds MG, et al. Phylogenetic and ecologic perspectives of a monkeypox outbreak, southern Sudan, 2005. Emerg Infect Dis. 2013; 19:237-45. http://dx.doi.org/10.3201/eid1902.121220

7. Kulesh DA, Loveless BM, Norwood D, Garrison J, Whitehouse CA, Hartmann C, et al. Monkeypox virus detection in rodents using real-time $3^{\prime}$-minor groove binder TaqMan 
assays on the Roche LightCycler. Lab Invest. 2004;84:1200-8. http://dx.doi.org/10.1038/labinvest.3700143

8. Hammarlund E, Lewis MW, Carter SV, Amanna I, Hansen SG, Strelow LI, et al. Multiple diagnostic techniques identify previously vaccinated individuals with protective immunity against monkeypox. Nat Med. 2005;11:1005-11. http://dx.doi.org/10.1038/nm1273

9. Lopera JG, Falendysz EA, Rocke TE, Osorio JE. Attenuation of monkeypox virus by deletion of genomic regions. Virology. 2015;475:129-38. http://dx.doi.org/10.1016/j.virol.2014.11.009

10. Peters JC. An epizootic of monkey pox at Rotterdam Zoo. Int Zoo Yearb. 1966;6:274-5. http://dx.doi.org/10.1111/j.1748-1090.1966. tb01794.x

Address for correspondence: Wenjie Tan, Chinese Center for Disease Control and Prevention, 155 Changbai Rd, Changping District, Beijing 102206, China; email: tanwj28@163.com

\section{Leishmaniasis in Norway Rats in Sewers, Barcelona, Spain}

\author{
Maria Teresa Galán-Puchades, \\ Mercedes Gómez-Samblás, \\ Jose M. Suárez-Morán, Antonio Osuna, \\ Joan Sanxis-Furió, Jordi Pascual, \\ Rubén Bueno-Marí, Sandra Franco, \\ Víctor Peracho, Tomás Montalvo, Màrius V. Fuentes
}

Author affiliations: Universitat de València, Burjassot-Valencia, Spain (M.T. Galán-Puchades, J. Sanxis-Furió, M.V. Fuentes); Universidad de Granada, Granada, Spain (M. Gómez-Samblás, J.M. Suárez-Morán, A. Osuna); Laboratorios Lokímica, Catarroja-Valencia, Spain (J. Sanxis-Furió, R. Bueno-Marí); Agència de Salut Pública de Barcelona, Barcelona, Spain (J. Pascual, S. Franco, V. Peracho, T. Montalvo); CIBER Epidemiology and Public Health, Barcelona (T. Montalvo)

DOI: https://doi.org/10.3201/eid2506.181027

We detected Leishmania infantum in 98 Norway rats (Rattus norvegicus) trapped in parks and sewers of Barcelona, Spain. The 84 rats from the sewers showed a prevalence of $33.3 \%$ and up to 2,272 estimated parasites. These results, in the most abundant potential reservoir in cities, is of public health concern.

$\mathrm{C}$ Tanine and human leishmaniasis caused by Leishmania infantum is considered an emerging disease in the Mediterranean basin (1). In addition to dogs, several wild mammals have been found infected by L. infantum in rural environments in Europe (2). With regard to the epidemiologic factors promoting infection with Leishmania, the appearance of new animal reservoirs besides dogs has been highlighted (1). In this context, only a few studies examine the possible reservoir role of synanthropic animals in cities, where the role of certain domestic mammals has been analyzed exclusively (1).

Because human leishmaniasis is endemic in Barcelona, Spain (3), we investigated and quantified the presence of $L$. infantum in an urban population of the Norway rat, Rattus norvegicus, using a highly sensitive quantitative PCR (qPCR) method for Leishmania DNA detection. Rat leishmaniasis could complicate the epidemiologic situation of human and canine leishmaniasis, considering that the Norway rat is the most widespread mammal in the world after humans and also the most abundant animal in cities.

We trapped 98 Norway rats, 84 in the sewage system and 14 in parks, during the winter of 2016-17 in a rodent surveillance and control program in Barcelona (permission no. SF/044 obtained from the regional government of Catalonia). We treated the rats according to Directive 2010/63/EU of the European Parliament and Council decision of September 22, 2010. We obtained DNA from $10 \mathrm{mg}$ of spleen using the Purification of Total DNA Kit (QIAGEN, https://www.qiagen.com), following the manufacturer's instructions. We processed the samples whose DNA concentration was too low with the extraction kit by the phenol-chloroform-isoamyl $(25: 24: 1)$ DNA extraction technique. We quantified the parasite DNA by qPCR using Taqman probe with Fam fluorochrome (4).

Only 1 rat $(7.1 \%)$ captured in the parks tested positive for $L$. infantum. However, rats captured in the sewage system showed a $33.3 \%$ prevalence $(28 / 84)$ of $L$. infantum infection. The estimated number of parasites in the positive samples from spleens varied considerably, ranging from 0.28 to $>2,200$ (Table). Histologic sections of positive spleens were stained by Giemsa and by the streptavidin-biotin peroxidase complex immunohistochemical method (5) (Appendix Figure, https://wwwnc.cdc.gov/ EID/article/25/6/18-1027-App1.pdf).

The low number of infected Norway rats found in Mediterranean countries in Europe so far has led to the species being categorized as an incidental host, capable of becoming infected but considered irrelevant to the longterm persistence of the disease (2,6-8). However, our study demonstrates the importance of the trapping site for finding a large Leishmania-infected rat population.

According to the World Health Organization, the incrimination of a particular mammal as a Leishmania reservoir must depend on an accumulation of evidence (9). First, the reservoir must be sufficiently abundant 